\title{
Existence and nonexistence of positive solutions for the fractional coupled system involving generalized $p$-Laplacian
}

Ying Wang ${ }^{1,2}$ and Jiqiang Jiang $3^{3^{*}}$

${ }^{\text {"Correspondence: }}$
jidihanxue@126.com
${ }^{3}$ School of Mathematical Sciences,
Qufu Normal University, Qufu,
Shandong 273165, People's
Republic of China
Full list of author information is
available at the end of the article

available at the end of the article

\begin{abstract}
In this article, we study a class of fractional coupled systems with Riemann-Stieltjes integral boundary conditions and generalized $p$-Laplacian which involves two different parameters. Based on the Guo-Krasnosel'skii fixed point theorem, some new results on the existence and nonexistence of positive solutions for the fractional system are received, the impact of the two different parameters on the existence and nonexistence of positive solutions is also investigated. An example is then given to illuminate the application of the main results.
\end{abstract}

MSC: $26 \mathrm{~A} 33 ; 34 \mathrm{~B} 18$

Keywords: positive solutions; fractional coupled system; Riemann-Stieltjes integral conditions; generalized $p$-Laplacian operator

\section{Introduction}

In this paper, our main research is the existence and nonexistence of positive solutions for the following fractional coupled system with generalized $p$-Laplacian involving RiemannStieltjes integral conditions.

$$
\begin{cases}D_{0^{+}}^{\beta_{1}}\left(\phi\left(D_{0^{+}}^{\alpha_{1}} u(t)\right)\right)+\lambda_{1} f_{1}(t, u(t), v(t))=0, & \\ D_{0^{+}}^{\beta_{2}}\left(\phi\left(D_{0^{+}}^{\alpha_{2}} v(t)\right)\right)+\lambda_{2} f_{2}(t, u(t), v(t))=0, \quad 0<t<1, \\ u(0)=u^{\prime}(0)=\cdots u^{(n-2)}=0, \quad \phi\left(D_{0^{+}}^{\alpha_{1}} u(0)\right)=\left(\phi\left(D_{0^{+}}^{\alpha_{1}} u(1)\right)\right)^{\prime}=0, \\ v(0)=v^{\prime}(0)=\cdots v^{(m-2)}=0, \quad \phi\left(D_{0^{+}}^{\alpha_{2}} v(0)\right)=\left(\phi\left(D_{0^{+}}^{\alpha_{2}} v(1)\right)\right)^{\prime}=0, \\ u(1)=\mu_{1} \int_{0}^{1} g_{1}(s) v(s) d A_{1}(s), \quad v(1)=\mu_{2} \int_{0}^{1} g_{2}(s) u(s) d A_{2}(s),\end{cases}
$$

where $\lambda_{i}>0(i=1,2)$ is a parameter, $1<\beta_{i} \leq 2, n-1<\alpha_{1} \leq n, m-1<\alpha_{2} \leq m, n, m \geq 2$, $D_{0^{+}}^{\alpha_{i}}, D_{0^{+}}^{\beta_{i}}$ are the standard Riemann-Liouville derivatives. $\mu_{i}>0$ is a constant, $g_{i}:(0,1) \rightarrow$ $[0,+\infty)$ is continuous with $g_{i} \in L^{1}(0,1), A_{i}$ is right continuous on $[0,1)$, left continuous at $t=1$, and nondecreasing on $[0,1], A_{i}(0)=0, \int_{0}^{1} x(s) d A_{i}(s)$ denotes the Riemann-Stieltjes integrals of $x$ with respect to $A_{i}, \phi$ is a generalized $p$-Laplacian operator and satisfies the following condition $\left(\mathbf{H}_{0}\right)$.

The positive solution $(u, v)$ of system (1) means that $(u, v) \in C[0,1] \times C[0,1],(u, v)$ satisfies system (1) and $u(t)>0, v(t)>0$ for all $t \in(0,1]$.

(c) The Author(s) 2017. This article is distributed under the terms of the Creative Commons Attribution 4.0 International License (http://creativecommons.org/licenses/by/4.0/), which permits unrestricted use, distribution, and reproduction in any medium, provided you give appropriate credit to the original author(s) and the source, provide a link to the Creative Commons license, and indicate if changes were made. 
$\left(\mathbf{H}_{0}\right) \phi: \mathbb{R} \rightarrow \mathbb{R}$ is an odd, increasing homeomorphism, and there exist two increasing homeomorphisms $\psi_{1}, \psi_{2}:(0,+\infty) \rightarrow(0,+\infty)$ such that

$$
\psi_{1}(x) \phi(y) \leq \phi(x y) \leq \psi_{2}(x) \phi(y), \quad x, y>0
$$

Moreover, $\phi, \phi^{-1} \in C^{1}(\mathbb{R})$, where $\phi^{-1}$ denotes the inverse of $\phi$ and $\mathbb{R}=(-\infty,+\infty)$.

Lemma 1.1 ([1]) Assume that $\left(\mathbf{H}_{0}\right)$ holds. Then

$$
\psi_{2}^{-1}(x) y \leq \phi^{-1}(x \phi(y)) \leq \psi_{1}^{-1}(x) y, \quad x, y>0
$$

For $\phi$ satisfying $\left(\mathbf{H}_{0}\right)$, we call it a generalized $p$-Laplacian operator, it contains two important special cases: $\phi(u)=u$ and $\phi(u)=|u|^{p-2} u(p>1)$ (see [1]). Many researchers have studied the existence of positive solutions for two above cases due to their great application background (see [2-15]). Combined with the fractional calculus, the application of the above two kinds of special circumstances becomes more extensive and practical. For the sake of considering the turbulent flow in a porous medium, the governing equation

$$
\frac{\partial}{\partial x}\left(\frac{\partial u^{m}}{\partial x}\left|\frac{\partial u^{m}}{\partial x}\right|^{p-1}\right)=g\left(t, u, \frac{\partial u}{\partial t}\right), \quad m \leq 2, \frac{1}{2} \leq p \leq 1
$$

was presented by Leibenson (see [2]). If $p=1, m>0$, it is used as a nonlinear model for the dispersion of animals and insects (see [3]).

In [4], Lu et al. studied the existence of positive solution for the fractional boundary value problem with a $p$-Laplacian operator:

$$
\left\{\begin{array}{l}
D_{0^{+}}^{\beta}\left(\phi_{p}\left(D_{0^{+}}^{\alpha} u(t)\right)\right)=f(t, u(t)), \quad 0<t<1, \\
u(0)=u^{\prime}(0)=u(1)=0, \quad D_{0^{+}}^{\alpha} u(0)=D_{0^{+}}^{\alpha} u(1)=0,
\end{array}\right.
$$

where $2<\alpha \leq 3,1<\beta \leq 2, D_{0^{+}}^{\alpha}, D_{0^{+}}^{\beta}$ are the standard Riemann-Liouville fractional derivatives, $\phi_{p}(s)=|s|^{p-2} s, p>1, \phi_{p}^{-1}=\phi_{q}, \frac{1}{p}+\frac{1}{q}=1, f:[0,1] \times[0,+\infty) \rightarrow[0,+\infty)$ is a continuous function. By the properties of Green's function and the Guo-Krasnosel'skii fixed point theorem, some results on the existence of positive solutions are obtained.

In [5], Wang et al. investigated the same equation as (3) for $1<\alpha \leq 2,0<\beta \leq 1$, with boundary value condition $u(0)=0, D_{0^{+}}^{\alpha} u(0)=0, u(1)=a u(\xi)$, where $0 \leq a \leq 1,0<\xi<1$, $f:[0,1] \times[0,+\infty) \rightarrow[0,+\infty)$ is a continuous function. Through the application of the Guo-Krasnosel'skii fixed point theorem and the Leggett-Williams theorem, sufficient conditions for the existence of positive solutions are received.

In system (1), $\int_{0}^{1} g_{1}(s) v(s) d A_{1}(s), \int_{0}^{1} g_{2}(s) u(s) d A_{2}(s)$ denote the Riemann-Stieltjes integrals, and $A_{i}$ is a function of bounded variation, which implies that $d A_{i}$ can be a signed measure. Then, a multipoint boundary value problem and an integral boundary value problem are included in our study, that is to say, system (1) includes more generalized 
boundary value conditions. Henderson and Luca in [16] considered the following system:

$$
\begin{cases}D_{0^{+}}^{\alpha_{1}} u(t)+\lambda_{1} f_{1}(t, u(t), v(t))=0, & \\ D_{0^{+}}^{\alpha_{2}} v(t)+\lambda_{2} f_{2}(t, u(t), v(t))=0, & 0<t<1, \\ u(0)=u^{\prime}(0)=\cdots u^{(n-2)}=0, & u(1)=\sum_{i=1}^{p} a_{i} u\left(\xi_{i}\right), \\ v(0)=v^{\prime}(0)=\cdots v^{(m-2)}=0, & v(1)=\sum_{i=1}^{q} b_{i} v\left(\eta_{i}\right),\end{cases}
$$

where $\lambda_{i}>0(i=1,2)$ is a parameter, $n-1<\alpha_{1} \leq n, m-1<\alpha_{2} \leq m, n, m \geq 2, D_{0^{+}}^{\alpha_{i}}, D_{0^{+}}^{\beta_{i}}$ are the standard Riemann-Liouville derivatives. $a_{i}>0, b_{i}>0$ are constants, $f_{i}:[0,1] \times$ $[0,+\infty) \times[0,+\infty) \rightarrow[0,+\infty)$ is a continuous function. By the Guo-Krasnosel'skii fixed point theorem, the authors in [16] got the existence of positive solutions on system (4). System (4) with uncoupled and coupled multi-point boundary value conditions

$$
\begin{aligned}
& \begin{cases}u(0)=u^{\prime}(0)=\cdots u^{(n-2)}=0, & u(1)=\mu_{1} \int_{0}^{1} u(s) d A_{1}(s), \\
v(0)=v^{\prime}(0)=\cdots v^{(m-2)}=0, & v(1)=\mu_{2} \int_{0}^{1} v(s) d A_{2}(s),\end{cases} \\
& \begin{cases}u(0)=u^{\prime}(0)=\cdots u^{(n-2)}=0, & u(1)=\mu_{1} \int_{0}^{1} v(s) d A_{1}(s), \\
v(0)=v^{\prime}(0)=\cdots v^{(m-2)}=0, & v(1)=\mu_{2} \int_{0}^{1} u(s) d A_{2}(s)\end{cases}
\end{aligned}
$$

has been studied in many papers, where $\mu_{i}>0$ is a constant, for $\mu_{i}=1$ as an exceptional case ( see [17-23] and the references therein). However, these articles only study the existence of positive solutions for the system, and do not relate to the nonexistence of positive solutions.

Up to now, coupled boundary value conditions for a fractional differential system with generalized p-Laplacian like system (1) have seldom been considered when $\lambda_{1}, \lambda_{2}$ are different. Motivated by the results mentioned above, in this paper, we obtain several new existence and nonexistence results for positive solutions in terms of different values of the parameter $\lambda_{i}$ by using the properties of Green's function and the Guo-Krasnosel'skii fixed point theorem on cone. Especially, paying attention to the nonlinear operator $D_{0^{+}}^{\beta}\left(\phi\left(D_{0^{+}}^{\alpha}\right)\right)$ with the discussion in (1), we can convert it to the linear operator $D_{0^{+}}^{\beta} D_{0^{+}}^{\alpha}$, if $\phi(u)=u$, and the additive index law

$$
D_{0^{+}}^{\beta} D_{0^{+}}^{\alpha} u(t)=D_{0^{+}}^{\alpha+\beta} u(t)
$$

holds under some reasonable constraints on the function $u$ (see [24]). Therefore, our article promotes, includes and improves the previous results in a certain degree.

\section{Preliminaries and lemmas}

For convenience of the reader, we present some necessary definitions about fractional calculus theory.

Definition 2.1 ([24, 25]) Let $\alpha>0$ and $u$ be piecewise continuous on $(0,+\infty)$ and integrable on any finite subinterval of $[0,+\infty)$. Then, for $t>0$, we call

$$
I_{0^{+}}^{\alpha} u(t)=\frac{1}{\Gamma(\alpha)} \int_{0}^{t}(t-s)^{\alpha-1} u(s) d s,
$$

the Riemann-Liouville fractional integral of $u$ of order $\alpha$. 
Definition $2.2([24,25])$ The Riemann-Liouville fractional derivative of order $\alpha>0$, $n-1 \leq \alpha<n, n \in \mathbb{N}$, is defined as

$$
D_{0^{+}}^{\alpha} u(t)=\frac{1}{\Gamma(n-\alpha)}\left(\frac{d}{d t}\right)^{n} \int_{0}^{t}(t-s)^{n-\alpha-1} u(s) d s,
$$

where $\mathbb{N}$ denotes the natural number set, the function $u(t)$ is $n$ times continuously differentiable on $[0,+\infty)$.

Lemma 2.1 $([24,25])$ Let $\alpha>0$, if the fractional derivatives $D_{0^{+}}^{\alpha-1} u(t)$ and $D_{0^{+}}^{\alpha} u(t)$ are continuous on $[0,+\infty)$, then

$$
I_{0^{+}}^{\alpha} D_{0^{+}}^{\alpha} u(t)=u(t)+c_{1} t^{\alpha-1}+c_{2} t^{\alpha-2}+\cdots+c_{n} t^{\alpha-n},
$$

where $c_{1}, c_{2}, \ldots, c_{n} \in(-\infty,+\infty), n$ is the smallest integer greater than or equal to $\alpha$.

Similarly to the proof in [22], it enables us to obtain the following Lemmas 2.2, 2.3 and Remark 2.1.

Lemma 2.2 Assume that the following condition $\left(\mathbf{H}_{1}\right)$ holds.

$\left(\mathbf{H}_{1}\right)$

$$
\begin{aligned}
& k_{1}=\int_{0}^{1} g_{1}(t) t^{\alpha_{2}-1} d A_{1}(t)>0, \quad k_{2}=\int_{0}^{1} g_{2}(t) t^{\alpha_{1}-1} d A_{2}(t)>0, \\
& 1-\mu_{1} \mu_{2} k_{1} k_{2}>0 .
\end{aligned}
$$

Let $h_{i} \in C(0,1) \cap L(0,1)(i=1,2)$, then the system with the coupled boundary conditions

$$
\left\{\begin{array}{l}
D_{0^{+}}^{\alpha_{1}} u(t)+h_{1}(t)=0, \quad D_{0^{+}}^{\alpha_{2}} v(t)+h_{2}(t)=0, \quad 0<t<1, \\
u(0)=u^{\prime}(0)=\cdots u^{(n-2)}=0, \quad u(1)=\mu_{1} \int_{0}^{1} g_{1}(s) v(s) d A_{1}(s), \\
v(0)=v^{\prime}(0)=\cdots v^{(n-2)}=0, \quad v(1)=\mu_{2} \int_{0}^{1} g_{2}(s) u(s) d A_{2}(s)
\end{array}\right.
$$

has a unique integral representation

$$
\left\{\begin{array}{l}
u(t)=\int_{0}^{1} K_{1}(t, s) h_{1}(s) d s+\int_{0}^{1} H_{1}(t, s) h_{2}(s) d s, \\
v(t)=\int_{0}^{1} K_{2}(t, s) h_{2}(s) d s+\int_{0}^{1} H_{2}(t, s) h_{1}(s) d s,
\end{array}\right.
$$

where

$$
\begin{aligned}
& K_{1}(t, s)=\frac{\mu_{1} \mu_{2} k_{1} t^{\alpha_{1}-1}}{1-\mu_{1} \mu_{2} k_{1} k_{2}} \int_{0}^{1} g_{2}(t) \bar{G}_{1}(t, s) d A_{2}(t)+\bar{G}_{1}(t, s), \\
& H_{1}(t, s)=\frac{\mu_{1} t^{\alpha_{1}-1}}{1-\mu_{1} \mu_{2} k_{1} k_{2}} \int_{0}^{1} g_{1}(t) \bar{G}_{2}(t, s) d A_{1}(t), \\
& K_{2}(t, s)=\frac{\mu_{2} \mu_{1} k_{2} t^{\alpha_{2}-1}}{1-\mu_{1} \mu_{2} k_{1} k_{2}} \int_{0}^{1} g_{1}(t) \bar{G}_{2}(t, s) d A_{1}(t)+\bar{G}_{2}(t, s), \\
& H_{2}(t, s)=\frac{\mu_{2} t^{\alpha_{2}-1}}{1-\mu_{1} \mu_{2} k_{1} k_{2}} \int_{0}^{1} g_{2}(t) \bar{G}_{1}(t, s) d A_{2}(t),
\end{aligned}
$$


and

$$
\bar{G}_{i}(t, s)=\frac{1}{\Gamma\left(\alpha_{i}\right)}\left\{\begin{array}{ll}
{[t(1-s)]^{\alpha_{i}-1}-(t-s)^{\alpha_{i}-1},} & 0 \leq s \leq t \leq 1 \\
{[t(1-s)]^{\alpha_{i}-1},} & 0 \leq t \leq s \leq 1
\end{array} \quad i=1,2\right.
$$

Lemma 2.3 For $t, s \in[0,1]$, the functions $K_{i}(t, s)$ and $H_{i}(t, s)(i=1,2)$ defined as $(7)$ satisfy

$$
\begin{aligned}
& K_{1}(t, s), H_{2}(t, s) \leq \rho s(1-s)^{\alpha_{1}-1}, \quad K_{2}(t, s), H_{1}(t, s) \leq \rho s(1-s)^{\alpha_{2}-1}, \\
& K_{1}(t, s), H_{1}(t, s) \leq \rho t^{\alpha_{1}-1}, \quad K_{2}(t, s), H_{2}(t, s) \leq \rho t^{\alpha_{2}-1}, \\
& K_{1}(t, s) \geq \varrho t^{\alpha_{1}-1} s(1-s)^{\alpha_{1}-1}, \quad H_{2}(t, s) \geq \varrho t^{\alpha_{2}-1} s(1-s)^{\alpha_{1}-1}, \\
& K_{2}(t, s) \geq \varrho t^{\alpha_{2}-1} s(1-s)^{\alpha_{2}-1}, \quad H_{1}(t, s) \geq \varrho t^{\alpha_{1}-1} s(1-s)^{\alpha_{2}-1},
\end{aligned}
$$

where

$$
\begin{aligned}
\rho= & \max \left\{\frac{1}{\Gamma\left(\alpha_{1}-1\right)}\left(\frac{\mu_{1} \mu_{2} k_{1}}{1-\mu_{1} \mu_{2} k_{1} k_{2}} \int_{0}^{1} g_{2}(t) d A_{2}(t)+1\right)\right. \\
& \frac{\mu_{1}}{\Gamma\left(\alpha_{2}-1\right)\left(1-\mu_{1} \mu_{2} k_{1} k_{2}\right)} \int_{0}^{1} g_{1}(t) d A_{1}(t), \\
& \frac{1}{\Gamma\left(\alpha_{2}-1\right)}\left(\frac{\mu_{2} \mu_{1} k_{2}}{1-\mu_{1} \mu_{2} k_{1} k_{2}} \int_{0}^{1} g_{1}(t) d A_{1}(t)+1\right) \\
& \left.\frac{\mu_{2}}{\Gamma\left(\alpha_{1}-1\right)\left(1-\mu_{1} \mu_{2} k_{1} k_{2}\right)} \int_{0}^{1} g_{2}(t) d A_{2}(t)\right\} \\
\varrho= & \frac{\mu_{1} \mu_{2} k_{1}}{\Gamma\left(\alpha_{1}\right)\left(1-\mu_{1} \mu_{2} k_{1} k_{2}\right)} \int_{0}^{1} g_{2}(t)(1-t) t^{\alpha_{1}-1} d A_{2}(t) \\
& \frac{\mu_{1}}{\Gamma\left(\alpha_{2}\right)\left(1-\mu_{1} \mu_{2} k_{1} k_{2}\right)} \int_{0}^{1} g_{1}(t)(1-t) t^{\alpha_{2}-1} d A_{1}(t) \\
& \frac{\mu_{2} \mu_{1} k_{2}}{\Gamma\left(\alpha_{2}\right)\left(1-\mu_{1} \mu_{2} k_{1} k_{2}\right)} \int_{0}^{1} g_{1}(t)(1-t) t^{\alpha_{2}-1} d A_{1}(t) \\
& \left.\frac{\mu_{2}}{\Gamma\left(\alpha_{1}\right)\left(1-\mu_{1} \mu_{2} k_{1} k_{2}\right)} \int_{0}^{1} g_{2}(t)(1-t) t^{\alpha_{1}-1} d A_{2}(t)\right\}
\end{aligned}
$$

Remark 2.1 From Lemma 2.3, for $t, \tilde{t}, s \in[0,1]$, we have

$$
\begin{array}{ll}
K_{i}(t, s) \geq \omega t^{\alpha_{i}-1} K_{i}(\widetilde{t}, s), & H_{i}(t, s) \geq \omega t^{\alpha_{i}-1} H_{i}(\widetilde{t}, s), \quad i=1,2, \\
K_{1}(t, s) \geq \omega t^{\alpha_{1}-1} H_{2}(\widetilde{t}, s), & H_{2}(t, s) \geq \omega t^{\alpha_{2}-1} K_{1}(\widetilde{t}, s), \\
K_{2}(t, s) \geq \omega t^{\alpha_{2}-1} H_{1}(\widetilde{t}, s), & H_{1}(t, s) \geq \omega t^{\alpha_{1}-1} K_{2}(\widetilde{t}, s),
\end{array}
$$

where $\omega=\frac{\varrho}{\rho}, \varrho, \rho$ are defined as in Lemma 2.3, $0<\omega<1$.

From Lemmas 2.1 and 2.2, we obtain the following Lemma 2.4. 
Lemma 2.4 Let $1<\beta_{i} \leq 2, n-1<\alpha_{1} \leq n, m-1<\alpha_{2} \leq m, h_{i} \in C(0,1) \cap L(0,1)(i=1,2)$, the following system of fractional differential equations

$$
\left\{\begin{array}{l}
D_{0^{+}}^{\beta_{1}}\left(\phi\left(D_{0^{+}}^{\alpha_{1}} u(t)\right)\right)+\lambda_{1} h_{1}(t)=0, \quad D_{0^{+}}^{\beta_{2}}\left(\phi\left(D_{0^{+}}^{\alpha_{2}} v(t)\right)\right)+\lambda_{2} h_{2}(t)=0, \quad 0<t<1, \\
u(0)=u^{\prime}(0)=\cdots u^{(n-2)}=0, \quad \phi\left(D_{0^{+}}^{\alpha_{1}} u(0)\right)=\left(\phi\left(D_{0^{+}}^{\alpha_{1}} u(1)\right)\right)^{\prime}=0, \\
v(0)=v^{\prime}(0)=\cdots v^{(m-2)}=0, \quad \phi\left(D_{0^{+}}^{\alpha_{2}} v(0)\right)=\left(\phi\left(D_{0^{+}}^{\alpha_{2}} v(1)\right)\right)^{\prime}=0, \\
u(1)=\mu_{1} \int_{0}^{1} v(s) d A_{1}(s), \quad v(1)=\mu_{2} \int_{0}^{1} u(s) d A_{2}(s)
\end{array}\right.
$$

has a unique integral representation

$$
\left\{\begin{aligned}
u(t)= & \int_{0}^{1} K_{1}(t, s) \phi^{-1}\left(\lambda_{1} \int_{0}^{1} G_{1}(s, \tau) h_{1}(\tau) d \tau\right) d s \\
& +\int_{0}^{1} H_{1}(t, s) \phi^{-1}\left(\lambda_{2} \int_{0}^{1} G_{2}(s, \tau) h_{2}(\tau) d \tau\right) d s, \\
v(t)= & \int_{0}^{1} K_{2}(t, s) \phi^{-1}\left(\lambda_{2} \int_{0}^{1} G_{2}(s, \tau) h_{2}(\tau) d \tau\right) d s \\
& +\int_{0}^{1} H_{2}(t, s) \phi^{-1}\left(\lambda_{1} \int_{0}^{1} G_{1}(s, \tau) h_{1}(\tau) d \tau\right) d s,
\end{aligned}\right.
$$

where

$$
G_{i}(s, \tau)=\frac{1}{\Gamma\left(\beta_{i}\right)}\left\{\begin{array}{ll}
s[s(1-\tau)]^{\beta_{i}-2}-(s-\tau)^{\beta_{i}-1}, & 0 \leq \tau \leq s \leq 1, \\
s[s(1-\tau)]^{\beta_{i}-2}, & 0 \leq s \leq \tau \leq 1,
\end{array} \quad i=1,2 .\right.
$$

Lemma $2.5([26])$ The function $G_{i}(s, \tau)$ defined as $(8)$ is continuous on $[0,1] \times[0,1]$, and for $s, \tau \in[0,1], G_{i}(s, \tau)$ satisfies
(1) $G_{i}(s, \tau) \geq 0$;
(2) $\quad G_{i}(s, \tau) \leq G_{i}(\tau, \tau)$;
(3) $\quad G_{i}(s, \tau) \geq s^{\beta_{i}-1} G_{i}(1, \tau)$.

In the rest of the paper, we always suppose that the following assumption holds:

$\left(\mathbf{H}_{2}\right) f_{i}:[0,1] \times[0,+\infty) \times[0,+\infty) \rightarrow[0,+\infty)$ is continuous.

Let $X=C[0,1] \times C[0,1]$, then $X$ is a Banach space with the norm

$$
\|(u, v)\|=\max \{\|u\|,\|v\|\}, \quad\|u\|=\max _{t \in[0,1]}|u(t)|, \quad\|v\|=\max _{t \in[0,1]}|v(t)| .
$$

Denote

$$
K=\left\{(u, v) \in X: u(t) \geq \omega t^{\alpha_{1}-1}\|(u, v)\|, v(t) \geq \omega t^{\alpha_{2}-1}\|(u, v)\|, t \in[0,1]\right\}
$$

where $\omega$ is defined as Remark 2.1. It is easy to see that $K$ is a positive cone in $X$. Under the above conditions $\left(\mathbf{H}_{0}\right)\left(\mathbf{H}_{1}\right)\left(\mathbf{H}_{2}\right)$, for any $(u, v) \in K$, we can define an integral operator $T: K \rightarrow X$ by

$$
T(u, v)(t)=\left(T_{1}(u, v)(t), T_{2}(u, v)(t)\right), \quad 0 \leq t \leq 1,
$$




$$
\begin{aligned}
T_{1}(u, v)(t)= & \int_{0}^{1} K_{1}(t, s) \phi^{-1}\left(\lambda_{1} \int_{0}^{1} G_{1}(s, \tau) f_{1}(\tau, u(\tau), v(\tau)) d \tau\right) d s \\
& +\int_{0}^{1} H_{1}(t, s) \phi^{-1}\left(\lambda_{2} \int_{0}^{1} G_{2}(s, \tau) f_{2}(\tau, u(\tau), v(\tau)) d \tau\right) d s, \\
T_{2}(u, v)(t)= & \int_{0}^{1} K_{2}(t, s) \phi^{-1}\left(\lambda_{2} \int_{0}^{1} G_{2}(s, \tau) f_{2}(\tau, u(\tau), v(\tau)) d \tau\right) d s \\
& +\int_{0}^{1} H_{2}(t, s) \phi^{-1}\left(\lambda_{1} \int_{0}^{1} G_{1}(s, \tau) f_{1}(\tau, u(\tau), v(\tau)) d \tau\right) d s,
\end{aligned}
$$

we know that $(u, v)$ is a positive solution of system (1) if and only if $(u, v)$ is a fixed point of $T$ in $K$.

Lemma 2.6 Assume that $\left(\mathbf{H}_{0}\right)\left(\mathbf{H}_{1}\right)\left(\mathbf{H}_{2}\right)$ hold. Then $T: K \rightarrow K$ is a completely continuous operator.

Proof By the routine discussion, we know that $T: K \rightarrow X$ is well defined, so we only prove $T(K) \subseteq K$. For any $(u, v) \in K, 0 \leq t, \tilde{t} \leq 1$, by Remark 2.1, we have

$$
\begin{aligned}
T_{1}(u, v)(t)= & \int_{0}^{1} K_{1}(t, s) \phi^{-1}\left(\lambda_{1} \int_{0}^{1} G_{1}(s, \tau) f_{1}(\tau, u(\tau), v(\tau)) d \tau\right) d s \\
& +\int_{0}^{1} H_{1}(t, s) \phi^{-1}\left(\lambda_{2} \int_{0}^{1} G_{2}(s, \tau) f_{2}(\tau, u(\tau), v(\tau)) d \tau\right) d s \\
\geq & \int_{0}^{1} \omega t^{\alpha_{1}-1} K_{1}(\widetilde{t}, s) \phi^{-1}\left(\lambda_{1} \int_{0}^{1} G_{1}(s, \tau) f_{1}(\tau, u(\tau), v(\tau)) d \tau\right) d s \\
& +\int_{0}^{1} \omega t^{\alpha_{1}-1} H_{1}(\widetilde{t}, s) \phi^{-1}\left(\lambda_{2} \int_{0}^{1} G_{2}(s, \tau) f_{2}(\tau, u(\tau), v(\tau)) d \tau\right) d s \\
\geq & \omega t^{\alpha_{1}-1}\left(\int_{0}^{1} K_{1}(\widetilde{t}, s) \phi^{-1}\left(\lambda_{1} \int_{0}^{1} G_{1}(s, \tau) f_{1}(\tau, u(\tau), v(\tau)) d \tau\right) d s\right. \\
& \left.+\int_{0}^{1} H_{1}(\widetilde{t}, s) \phi^{-1}\left(\lambda_{2} \int_{0}^{1} G_{2}(s, \tau) f_{2}(\tau, u(\tau), v(\tau)) d \tau\right) d s\right) \\
\geq & \omega t^{\alpha_{1}-1} T_{1}(u, v)(\widetilde{t}) .
\end{aligned}
$$

On the other hand,

$$
\begin{aligned}
T_{1}(u, v)(t) \geq & \int_{0}^{1} \omega t^{\alpha_{1}-1} H_{2}(\widetilde{t}, s) \phi^{-1}\left(\lambda_{1} \int_{0}^{1} G_{1}(s, \tau) f_{1}(\tau, u(\tau), v(\tau)) d \tau\right) d s \\
& +\int_{0}^{1} \omega t^{\alpha_{1}-1} K_{2}(\widetilde{t}, s) \phi^{-1}\left(\lambda_{2} \int_{0}^{1} G_{2}(s, \tau) f_{2}(\tau, u(\tau), v(\tau)) d \tau\right) d s \\
\geq & \omega t^{\alpha_{1}-1}\left(\int_{0}^{1} H_{2}(\widetilde{t}, s) \phi^{-1}\left(\lambda_{1} \int_{0}^{1} G_{1}(s, \tau) f_{1}(\tau, u(\tau), v(\tau)) d \tau\right) d s\right. \\
& \left.+\int_{0}^{1} K_{2}(\tilde{t}, s) \phi^{-1}\left(\lambda_{2} \int_{0}^{1} G_{2}(s, \tau) f_{2}(\tau, u(\tau), v(\tau)) d \tau\right) d s\right) \\
\geq & \omega t^{\alpha_{1}-1} T_{2}(u, v)(\widetilde{t}) .
\end{aligned}
$$


Then we have

$$
T_{1}(u, v)(t) \geq \omega t^{\alpha_{1}-1}\left\|T_{1}(u, v)\right\|, \quad T_{1}(u, v)(t) \geq \omega t^{\alpha_{1}-1}\left\|T_{2}(u, v)\right\|,
$$

i.e.,

$$
T_{1}(u, v)(t) \geq \omega t^{\alpha_{1}-1}\left\|\left(T_{1}(u, v), T_{2}(u, v)\right)\right\| .
$$

In the same way as (11) and (12), we can prove that

$$
T_{2}(u, v)(t) \geq \omega t^{\alpha_{2}-1}\left\|\left(T_{1}(u, v), T_{2}(u, v)\right)\right\| .
$$

Therefore, we have $T(K) \subseteq K$.

According to the Ascoli-Arzela theorem, we can easily get that $T: K \rightarrow K$ is completely continuous. The proof is completed.

In order to obtain the existence of the positive solutions of system (1), we will use the following cone compression and expansion fixed point theorem.

Lemma 2.7 ([27]) Let $P$ be a positive cone in a Banach space $E, \Omega_{1}$ and $\Omega_{2}$ are bounded open sets in $E, \theta \in \Omega_{1}, \bar{\Omega}_{1} \subset \Omega_{2}, A: P \cap \bar{\Omega}_{2} \backslash \Omega_{1} \rightarrow P$ is a completely continuous operator. If the following conditions are satisfied:

$$
\|A x\| \leq\|x\|, \quad \forall x \in P \cap \partial \Omega_{1}, \quad\|A x\| \geq\|x\|, \quad \forall x \in P \cap \partial \Omega_{2},
$$

or

$$
\|A x\| \geq\|x\|, \quad \forall x \in P \cap \partial \Omega_{1}, \quad\|A x\| \leq\|x\|, \quad \forall x \in P \cap \partial \Omega_{2},
$$

then $A$ has at least one fixed point in $P \cap\left(\bar{\Omega}_{2} \backslash \Omega_{1}\right)$.

\section{Main results}

Denote

$$
\begin{aligned}
& f_{10}=\liminf _{x \rightarrow 0^{+}} \inf _{\substack{t \in[a, b] \subset(0,1) \\
y \in[0,+\infty)}} \frac{f_{1}(t, x, y)}{\phi(x)}, \quad f_{1}^{0}=\limsup _{x \rightarrow 0^{+}} \sup _{\substack{t \in[0,1] \\
y \in[0,+\infty)}} \frac{f_{1}(t, x, y)}{\phi(x)}, \\
& f_{20}=\liminf _{y \rightarrow 0^{+}} \inf _{\substack{t \in[a, b] \subset(0,1) \\
x \in[0,+\infty)}} \frac{f_{2}(t, x, y)}{\phi(y)}, \quad f_{2}^{0}=\limsup _{y \rightarrow 0^{+}} \sup _{\substack{t \in[0,1] \\
x \in[0,+\infty)}} \frac{f_{2}(t, x, y)}{\phi(y)}, \\
& f_{1 \infty}=\liminf _{x \rightarrow+\infty} \inf _{\substack{t \in[a, b] \subset(0,1) \\
y \in[0,+\infty)}} \frac{f_{1}(t, x, y)}{\phi(x)}, \quad f_{1}^{\infty}=\limsup _{x \rightarrow+\infty} \sup _{\substack{t \in[0,1] \\
y \in[0,+\infty)}} \frac{f_{1}(t, x, y)}{\phi(x)}, \\
& f_{2 \infty}=\liminf _{y \rightarrow+\infty} \inf _{\substack{t \in[a, b] \subset(0,1) \\
x \in[0,+\infty)}} \frac{f_{2}(t, x, y)}{\phi(y)}, \quad f_{2}^{\infty}=\limsup _{y \rightarrow+\infty} \sup _{\substack{t \in[0,1] \\
x \in[0,+\infty)}} \frac{f_{2}(t, x, y)}{\phi(y)}, \\
& L_{1}=\max \left\{2 \rho \varphi_{1}^{-1}\left(\int_{0}^{1} G_{1}(\tau, \tau) d \tau\right), 2 \rho \varphi_{1}^{-1}\left(\int_{0}^{1} G_{2}(\tau, \tau) d \tau\right)\right\},
\end{aligned}
$$




$$
\begin{aligned}
L_{2}= & \min \left\{2 \varrho \omega \theta^{2} \int_{0}^{1} \varphi_{2}^{-1}\left(s^{\beta_{1}-1}\right) s^{\beta_{1}-1} \varphi_{2}^{-1}\left(\int_{0}^{1} G_{1}(1, \tau) d \tau\right) d s,\right. \\
& \left.2 \varrho \omega \theta^{2} \int_{0}^{1} \varphi_{2}^{-1}\left(s^{\beta_{2}-1}\right) s^{\beta_{2}-1} \varphi_{2}^{-1}\left(\int_{0}^{1} G_{2}(1, \tau) d \tau\right) d s\right\}, \\
L_{3}= & \min \left\{2 \varrho \theta \int_{0}^{1} \varphi_{2}^{-1}\left(s^{\beta_{1}-1}\right) s(1-s)^{\alpha_{1}-1} \varphi_{2}^{-1}\left(\int_{0}^{1} G_{1}(1, \tau) d \tau\right) d s,\right. \\
& \left.2 \varrho \theta \int_{0}^{1} \varphi_{2}^{-1}\left(s^{\beta_{2}-1}\right) s(1-s)^{\alpha_{2}-1} \varphi_{2}^{-1}\left(\int_{0}^{1} G_{2}(1, \tau) d \tau\right) d s\right\}, \quad \theta=\min _{t \in[a, b]}\left\{t^{\alpha_{1}-1}, t^{\alpha_{2}-1}\right\} .
\end{aligned}
$$

\subsection{Existence of system (1)}

Theorem 3.1 Assume that $\left(\mathbf{H}_{0}\right)\left(\mathbf{H}_{1}\right)\left(\mathbf{H}_{2}\right)$ hold and $f_{i \infty} \varphi_{1}\left(L_{1}^{-1}\right)>f_{i}^{0} \varphi_{2}\left(L_{2}^{-1}\right)$, then system (1) has at least one positive solution for

$$
\lambda_{i} \in\left(\frac{\varphi_{2}\left(L_{2}^{-1}\right)}{f_{i \infty}}, \frac{\varphi_{1}\left(L_{1}^{-1}\right)}{f_{i}^{0}}\right)
$$

where we impose $\frac{1}{f_{i \infty}}=0$ if $f_{i \infty}=+\infty$ and $\frac{1}{f_{i}^{0}}=+\infty$ iff $f_{i}^{0}=0(i=1,2)$.

Proof For any $\lambda_{i}$ satisfying (13), there exists $\varepsilon_{0}>0$ such that

$$
\frac{\varphi_{2}\left(L_{2}^{-1}\right)}{f_{i \infty}-\varepsilon_{0}} \leq \lambda_{i} \leq \frac{\varphi_{1}\left(L_{1}^{-1}\right)}{f_{i}^{0}+\varepsilon_{0}} .
$$

By the definition of $f_{i}^{0}$, there exists $r_{1}>0$ such that

$$
f_{i}(t, x, y) \leq\left(f_{i}^{0}+\varepsilon_{0}\right) \max \{\phi(x), \phi(y)\}, \quad 0 \leq x, y \leq r_{1}, t \in[0,1] .
$$

Let $K_{r_{1}}=\left\{(u, v) \in K:\|(u, v)\|<r_{1}\right\}$. For any $(u, v) \in \partial K_{r_{1}}, t \in[0,1]$, by the definition of $\|\cdot\|$, we know that

$$
\begin{aligned}
& u(t) \leq|u(t)| \leq\|u\| \leq\|(u, v)\| \leq r_{1}, \\
& v(t) \leq|v(t)| \leq\|v\| \leq\|(u, v)\| \leq r_{1}, \quad t \in[0,1] .
\end{aligned}
$$

Thus, for any $(u, v) \in \partial K_{r_{1}}$, by (15), (16) and $\left(\mathbf{H}_{0}\right)$, we have

$$
f_{i}(t, u(t), v(t)) \leq\left(f_{i}^{0}+\varepsilon_{0}\right) \phi\left(r_{1}\right), \quad t \in[0,1]
$$

Hence, for any $(u, v) \in \partial K_{r_{1}}$, by Lemmas 1.1, 2.3, 2.5 and (17), we conclude that

$$
\begin{aligned}
\left\|T_{1}(u, v)(t)\right\|= & \max _{t \in[0,1]} \mid \int_{0}^{1} K_{1}(t, s) \phi^{-1}\left(\lambda_{1} \int_{0}^{1} G_{1}(s, \tau) f_{1}(\tau, u(\tau), v(\tau)) d \tau\right) d s \\
& +\int_{0}^{1} H_{1}(t, s) \phi^{-1}\left(\lambda_{2} \int_{0}^{1} G_{2}(s, \tau) f_{2}(\tau, u(\tau), v(\tau)) d \tau\right) d s \mid \\
\leq & \max _{t \in[0,1]} \mid \int_{0}^{1} \rho t^{\alpha_{1}-1} \phi^{-1}\left(\lambda_{1} \int_{0}^{1} G_{1}(\tau, \tau)\left(f_{1}^{0}+\varepsilon_{0}\right) \phi\left(r_{1}\right) d \tau\right) d s \\
& +\int_{0}^{1} \rho t^{\alpha_{1}-1} \phi^{-1}\left(\lambda_{2} \int_{0}^{1} G_{2}(\tau, \tau)\left(f_{2}^{0}+\varepsilon_{0}\right) \phi\left(r_{1}\right) d \tau\right) d s \mid
\end{aligned}
$$




$$
\begin{aligned}
\leq & \rho r_{1} \varphi_{1}^{-1}\left(\lambda_{1}\left(f_{1}^{0}+\varepsilon_{0}\right)\right) \varphi_{1}^{-1}\left(\int_{0}^{1} G_{1}(\tau, \tau) d \tau\right) \\
& +\rho r_{1} \varphi_{1}^{-1}\left(\lambda_{2}\left(f_{2}^{0}+\varepsilon_{0}\right)\right) \varphi_{1}^{-1}\left(\int_{0}^{1} G_{2}(\tau, \tau) d \tau\right) \\
\leq & r_{1} \varphi_{1}^{-1}\left(\lambda_{1}\left(f_{1}^{0}+\varepsilon_{0}\right)\right) \frac{L_{1}}{2}+r_{1} \varphi_{1}^{-1}\left(\lambda_{2}\left(f_{2}^{0}+\varepsilon_{0}\right)\right) \frac{L_{1}}{2} \\
\leq & r_{1}=\|(u, v)\| .
\end{aligned}
$$

Similarly to (18), for any $(u, v) \in \partial K_{r_{1}}$, we also have

$$
\left\|T_{2}(u, v)\right\| \leq r_{1}=\|(u, v)\| .
$$

Consequently, we have

$$
\|T(u, v)\|=\max \left\{\left\|T_{1}(u, v)\right\|,\left\|T_{2}(u, v)\right\|\right\} \leq r_{1}=\|(u, v)\|, \quad(u, v) \in \partial K_{r_{1}} .
$$

On the other hand, by the definition of $f_{i \infty}$, there exist $r_{1}^{\prime}, r_{2}^{\prime}>0$ such that

$$
\begin{aligned}
& f_{1}(t, x, y) \geq\left(f_{1 \infty}-\varepsilon_{0}\right) \phi(x), \quad x \geq r^{\prime}, y \geq 0, t \in[a, b] \subset(0,1), \\
& f_{2}(t, x, y) \geq\left(f_{2 \infty}-\varepsilon_{0}\right) \phi(y), \quad y \geq r^{\prime}, x \geq 0, t \in[a, b] \subset(0,1) .
\end{aligned}
$$

Choose $r_{2}=\max \left\{\frac{r_{1}^{\prime}}{\omega \theta}, \frac{r_{2}^{\prime}}{\omega \theta}, 2 r_{1}\right\}$. Let $K_{r_{2}}=\left\{(u, v) \in K:\|(u, v)\|<r_{2}\right\}$. For any $(u, v) \in \partial K_{r_{2}}$, by the definition of $\|\cdot\|$, we have

$$
\begin{aligned}
& u(t) \geq \omega t^{\alpha_{1}-1}\|(u, v)\| \geq \omega \theta r_{2} \geq r_{1}^{\prime}, \\
& v(t) \geq \omega t^{\alpha_{2}-1}\|(u, v)\| \geq \omega \theta r_{2} \geq r_{2}^{\prime}, \quad t \in[a, b] \subset(0,1) .
\end{aligned}
$$

Thus, for any $(u, v) \in \partial K_{r_{2}}$, by (20), (21) and $\left(\mathbf{H}_{0}\right)$, we have

$$
\begin{array}{ll}
f_{1}(t, u(t), v(t)) \geq\left(f_{1 \infty}-\varepsilon_{0}\right) \phi(u(t)) \geq\left(f_{1 \infty}-\varepsilon_{0}\right) \phi\left(\omega \theta r_{2}\right), & t \in[a, b] \subset(0,1), \\
f_{2}(t, u(t), v(t)) \geq\left(f_{2 \infty}-\varepsilon_{0}\right) \phi(v(t)) \geq\left(f_{2 \infty}-\varepsilon_{0}\right) \phi\left(\omega \theta r_{2}\right), & t \in[a, b] \subset(0,1) .
\end{array}
$$

Hence, for any $(u, v) \in \partial K_{r_{2}}$, by Lemmas 1.1, 2.3, 2.5 and (22), we have

$$
\begin{aligned}
& \left\|T_{1}(u, v)(t)\right\| \\
& \quad \geq \min _{t \in[a, b]} \mid \int_{0}^{1} \varrho t^{\alpha_{1}-1} s(1-s)^{\alpha_{1}-1} \phi^{-1}\left(\lambda_{1} \int_{0}^{1} s^{\beta_{1}-1} G_{1}(1, \tau)\left(f_{1 \infty}-\varepsilon_{0}\right) \phi\left(\omega \theta r_{2}\right) d \tau\right) d s \\
& \quad+\int_{0}^{1} \varrho t^{\alpha_{1}-1} s(1-s)^{\alpha_{2}-1} \phi^{-1}\left(\lambda_{2} \int_{0}^{1} s^{\beta_{2}-1} G_{2}(1, \tau)\left(f_{2 \infty}-\varepsilon_{0}\right) \phi\left(\omega \theta r_{2}\right) d \tau\right) d s \mid \\
& \quad \geq \varrho \omega \theta^{2} r_{2} \int_{0}^{1} \varphi_{2}^{-1}\left(s^{\beta_{1}-1}\right) s^{\beta_{1}-1} \varphi_{2}^{-1}\left(\int_{0}^{1} G_{1}(1, \tau) d \tau\right) d s \psi_{2}^{-1}\left(\lambda_{1}\left(f_{1 \infty}-\varepsilon_{0}\right)\right) \\
& \quad+\varrho \omega \theta^{2} r_{2} \int_{0}^{1} \varphi_{2}^{-1}\left(s^{\beta_{2}-1}\right) s^{\beta_{2}-1} \varphi_{2}^{-1}\left(\int_{0}^{1} G_{2}(1, \tau) d \tau\right) d s \psi_{2}^{-1}\left(\lambda_{2}\left(f_{2 \infty}-\varepsilon_{0}\right)\right) \\
& \quad \geq r_{2} \varphi_{2}^{-1}\left(\lambda_{1}\left(f_{1 \infty}-\varepsilon_{0}\right)\right) \frac{L_{2}}{2}+r_{2} \varphi_{2}^{-1}\left(\lambda_{2}\left(f_{2 \infty}-\varepsilon_{0}\right)\right) \frac{L_{2}}{2} \\
& \quad \geq r_{2}=\|(u, v)\| .
\end{aligned}
$$


Therefore, we obtain

$$
\|T(u, v)\|=\max \left\{\left\|T_{1}(u, v)\right\|,\left\|T_{2}(u, v)\right\|\right\} \geq r_{2}=\|(u, v)\| \quad \text { for any }(u, v) \in \partial K_{r_{2}} .
$$

It follows from the above discussion, (18), (24), Lemmas 2.6 and 2.7 that, for any $\lambda_{i} \in$ $\left(\frac{\varphi_{2}\left(L_{2}^{-1}\right)}{f_{i \infty}}, \frac{\varphi_{1}\left(L_{1}^{-1}\right)}{f_{i}^{0}}\right), T$ has a fixed point $(u, v) \in \bar{K}_{r_{2}} \backslash K_{r_{1}}$, so system (1) has at least one positive solution $(u, v)$; moreover, $(u, v)$ satisfies $r_{1} \leq\|(u, v)\| \leq r_{2}$. The proof is completed.

Remark 3.1 From the proof of Theorem 3.1, if we choose

$$
\bar{L}_{2}=\varrho \omega \theta^{2} \int_{0}^{1} \varphi_{2}^{-1}\left(s^{\beta_{1}-1}\right) s^{\beta_{1}-1} \varphi_{2}^{-1}\left(\int_{0}^{1} G_{1}(1, \tau) d \tau\right) d s, \quad \theta=\min _{t \in[a, b]}\left\{t^{\alpha_{1}-1}, t^{\alpha_{2}-1}\right\},
$$

then for $\lambda_{1} \in\left(\frac{\varphi_{2}\left(\bar{L}_{2}^{-1}\right)}{f_{1 \infty}}, \frac{\varphi_{1}\left(L_{1}^{-1}\right)}{f_{1}^{0}}\right), \lambda_{2} \in\left(0, \frac{\varphi_{1}\left(L_{1}^{-1}\right)}{f_{2}^{0}}\right)$, the conclusion of Theorem 3.1 is valid.

Or we choose

$$
\widetilde{L}_{2}=\varrho \omega \theta^{2} \int_{0}^{1} \varphi_{2}^{-1}\left(s^{\beta_{2}-1}\right) s^{\beta_{2}-1} \varphi_{2}^{-1}\left(\int_{0}^{1} G_{2}(1, \tau) d \tau\right) d s, \quad \theta=\min _{t \in[a, b]}\left\{t^{\alpha_{1}-1}, t^{\alpha_{2}-1}\right\},
$$

then, for $\lambda_{1} \in\left(0, \frac{\varphi_{1}\left(L_{1}^{-1}\right)}{f_{1}^{0}}\right), \lambda_{2} \in\left(\frac{\varphi_{2}\left(\widetilde{L}_{2}^{-1}\right)}{f_{2 \infty}}, \frac{\varphi_{1}\left(L_{1}^{-1}\right)}{f_{2}^{0}}\right)$, the conclusion of Theorem 3.1 is valid.

Theorem 3.2 Assume that $\left(\mathbf{H}_{0}\right)\left(\mathbf{H}_{1}\right)\left(\mathbf{H}_{2}\right)$ hold and $f_{i 0} \varphi_{1}\left(L_{1}^{-1}\right)>f_{i}^{\infty} \varphi_{2}\left(L_{2}^{-1}\right)$, then system (1) has at least one positive solution for

$$
\lambda_{i} \in\left(\frac{\varphi_{2}\left(L_{2}^{-1}\right)}{f_{i 0}}, \frac{\varphi_{1}\left(L_{1}^{-1}\right)}{f_{i}^{\infty}}\right)
$$

where we impose $\frac{1}{f_{i 0}}=0$ if $f_{i 0}=+\infty$ and $\frac{1}{f_{i}^{\infty}}=+\infty$ if $f_{i}^{\infty}=0, i=1,2$.

The proof of Theorem 3.2 is similar to that of Theorem 3.1, and so we omit it.

Remark 3.2 Similar to Remark 3.1, if we choose $\bar{L}_{2}$ as (25), then for $\lambda_{1} \in\left(\frac{\varphi_{2}\left(\bar{L}_{2}^{-1}\right)}{f_{10}}, \frac{\varphi_{1}\left(L_{1}^{-1}\right)}{f_{1}^{\infty}}\right)$, $\lambda_{2} \in\left(0, \frac{\varphi_{1}\left(L_{1}^{-1}\right)}{f_{2}^{\infty}}\right)$, the conclusion of Theorem 3.2 is valid.

Or we choose $\widetilde{L}_{2}$ as $(26)$, then for $\lambda_{1} \in\left(0, \frac{\varphi_{1}\left(L_{1}^{-1}\right)}{f_{1}^{\infty}}\right), \lambda_{2} \in\left(\frac{\varphi_{2}\left(\widetilde{L}_{2}^{-1}\right)}{f_{20}}, \frac{\varphi_{1}\left(L_{1}^{-1}\right)}{f_{2}^{\infty}}\right)$, the conclusion of Theorem 3.2 is valid.

Theorem 3.3 Assume that $\left(\mathbf{H}_{0}\right)\left(\mathbf{H}_{1}\right)\left(\mathbf{H}_{2}\right)$ hold and there exist $R>r>0$ such that

$$
\lambda_{i} \min _{\substack{t \in[a, b] \subset(0,1) \\ \omega \theta r \leq x, y \leq r}} f_{i}(t, x, y) \geq \phi\left(\frac{r}{L_{3}}\right), \quad \lambda_{i} \max _{\substack{t \in[0,1] \\ 0 \leq x, y \leq R}} f_{i}(t, x, y) \leq \phi\left(\frac{R}{L_{1}}\right), \quad i=1,2 .
$$

Then system (1) has at least one positive solution $(u, v)$; moreover, $(u, v)$ satisfies $r \leq$ $\|(u, v)\| \leq R$.

Proof Set $K_{r}=\{(u, v) \in K:\|(u, v)\|<r\}$. For any $(u, v) \in \partial K_{r}$, by the definition of $\|\cdot\|$, we have

$$
\begin{aligned}
& \omega \theta r \leq \omega t^{\alpha_{1}-1} r=\omega t^{\alpha_{1}-1}\|(u, v)\| \leq u(t) \leq r, \\
& \omega t^{\alpha_{2}-1} r=\omega t^{\alpha_{2}-1}\|(u, v)\| \leq v(t) \leq r, \quad t \in[a, b] \subset(0,1) .
\end{aligned}
$$


Thus, for any $(u, v) \in \partial K_{r}$, by the first inequality of (27), we have

$$
\lambda_{i} \min _{\substack{t \in[a, b] \subset(0,1) \\ \omega \theta r \leq u(t), v(t) \leq r}} f_{i}(t, u(t), v(t)) \geq \phi\left(\frac{r}{L_{3}}\right), \quad i=1,2 .
$$

Hence, for any $(u, v) \in \partial K_{r}$, by Lemmas 1.1, 2.3, 2.5 and (28), we have

$$
\begin{aligned}
& \left\|T_{1}(u, v)(t)\right\| \\
& \geq \min _{t \in[a, b]} \mid \int_{0}^{1} \varrho t^{\alpha_{1}-1} s(1-s)^{\alpha_{1}-1} \phi^{-1}\left(\lambda_{1} \int_{0}^{1} s^{\beta_{1}-1} G_{1}(1, \tau) f_{1}(\tau, u(\tau), v(\tau)) d \tau\right) d s \\
& +\int_{0}^{1} \varrho t^{\alpha_{1}-1} s(1-s)^{\alpha_{2}-1} \phi^{-1}\left(\lambda_{2} \int_{0}^{1} s^{\beta_{2}-1} G_{2}(1, \tau) f_{2}(\tau, u(\tau), v(\tau)) d \tau\right) d s \mid \\
& \geq \varrho \theta \int_{0}^{1} s(1-s)^{\alpha_{1}-1} \phi^{-1}\left(\lambda_{1} \int_{0}^{1} s^{\beta_{1}-1} G_{1}(1, \tau) \min _{\substack{\tau \in[a, b] \subset(0,1) \\
\omega r \leq u(\tau), v(\tau) \leq r}} f_{1}(\tau, u(\tau), v(\tau)) d \tau\right) d s \\
& +\varrho \theta \int_{0}^{1} s(1-s)^{\alpha_{2}-1} \phi^{-1}\left(\lambda_{2} \int_{0}^{1} s^{\beta_{2}-1} G_{2}(1, \tau) \min _{\substack{\tau \in[a, b] \subset(0,1) \\
\omega r \leq u(\tau), v(\tau) \leq r}} f_{2}(\tau, u(\tau), v(\tau)) d \tau\right) d s \\
& \geq \phi^{-1}\left(\lambda_{1} \min _{\substack{\tau \in[a, b] \subset(0,1) \\
\omega r \leq u(\tau), v(\tau) \leq r}} f_{1}(\tau, u(\tau), v(\tau))\right) \frac{L_{3}}{2} \\
& +\phi^{-1}\left(\lambda_{2} \min _{\substack{\tau \in[a, b] \subset(0,1) \\
\omega r \leq u(\tau), v(\tau) \leq r}} f_{2}(\tau, u(\tau), v(\tau))\right) \frac{L_{3}}{2} \\
& \geq r=\|(u, v)\| \text {. }
\end{aligned}
$$

Therefore, we obtain

$$
\|T(u, v)\|=\max \left\{\left\|T_{1}(u, v)\right\|,\left\|T_{2}(u, v)\right\|\right\} \geq r=\|(u, v)\| \quad \text { for any }(u, v) \in \partial K_{r} .
$$

Choose $K_{R}=\{(u, v) \in K:\|(u, v)\|<R\}$. For any $(u, v) \in \partial K_{R}, t \in[0,1]$, by the definition of $\|\cdot\|$, we know that

$$
\begin{aligned}
& u(t) \leq|u(t)| \leq\|u\| \leq\|(u, v)\| \leq R, \\
& v(t) \leq|v(t)| \leq\|v\| \leq\|(u, v)\| \leq R, \quad t \in[0,1] .
\end{aligned}
$$

Thus, for any $(u, v) \in \partial K_{R}$, by the first inequality of (27) and (31), we have

$$
\lambda_{i} \max _{\substack{t \in[0,1] \\ 0 \leq u(t), v(t) \leq R}} f_{i}(t, u(t), v(t)) \leq \phi\left(\frac{R}{L_{1}}\right), \quad i=1,2 .
$$

Hence, for any $(u, v) \in \partial K_{R}$, by Lemmas 1.1, 2.3, 2.5 and (32), we can gain

$$
\begin{aligned}
\left\|T_{1}(u, v)(t)\right\| \leq & \max _{t \in[0,1]} \mid \int_{0}^{1} \rho t^{\alpha_{1}-1} \phi^{-1}\left(\lambda_{1} \int_{0}^{1} G_{1}(\tau, \tau) \max _{\substack{\tau \in[0,1] \\
0 \leq u(\tau), v(\tau) \leq R}} f_{1}(\tau, u(\tau), v(\tau)) d \tau\right) d s \\
& +\int_{0}^{1} \rho t^{\alpha_{1}-1} \phi^{-1}\left(\lambda_{2} \int_{0}^{1} G_{2}(\tau, \tau) \max _{\substack{\tau \in[0,1] \\
0 \leq u(\tau), \nu(\tau) \leq R}} f_{2}(\tau, u(\tau), v(\tau)) d \tau\right) d s \mid
\end{aligned}
$$




$$
\begin{aligned}
\leq & \phi^{-1}\left(\lambda_{1} \max _{\substack{\tau \in[0,1] \\
0 \leq u(\tau), v(\tau) \leq R}} f_{1}(\tau, u(\tau), v(\tau))\right) \frac{L_{1}}{2} \\
& +\phi_{1}^{-1}\left(\lambda_{2} \max _{\substack{\tau \in[0,1] \\
0 \leq u(\tau), v(\tau) \leq R}} f_{i}(\tau, u(\tau), v(\tau))\right) \frac{L_{1}}{2} \\
\leq & R=\|(u, v)\| .
\end{aligned}
$$

Similarly to (33), for any $(u, v) \in \partial K_{R}$, we also have

$$
\left\|T_{2}(u, v)\right\|<R=\|(u, v)\| .
$$

Consequently, we have

$$
\|T(u, v)\|=\max \left\{\left\|T_{1}(u, v)\right\|,\left\|T_{2}(u, v)\right\|\right\}<R=\|(u, v)\|, \quad(u, v) \in \partial K_{R} .
$$

It follows from the above discussion, (30), (34), Lemmas 2.6 and 2.7 that $T$ has a fixed point $(u, v) \in \bar{K}_{R} \backslash K_{r}$, so system (1) has at least one positive solution $(u, v)$; moreover, $(u, v)$ satisfies $r \leq\|(u, v)\| \leq R$. The proof is completed.

Remark 3.3 From the proof of Theorem 3.3, if we choose

$$
\begin{aligned}
\bar{L}_{3} & =\varrho \theta \int_{0}^{1} \varphi_{2}^{-1}\left(s^{\beta_{1}-1}\right) s(1-s)^{\alpha_{1}-1} \varphi_{2}^{-1}\left(\int_{0}^{1} G_{1}(1, \tau) d \tau\right) d s, \\
\theta & =\min _{t \in[a, b]}\left\{t^{\alpha_{1}-1}, t^{\alpha_{2}-1}\right\},
\end{aligned}
$$

then for

$$
\lambda_{1} \min _{\substack{t \in[a, b] \subset(0,1) \\ \omega r \leq x, y \leq r}} f_{1}(t, x, y) \geq \phi\left(\frac{r}{\bar{L}_{3}}\right), \quad \lambda_{i} \max _{\substack{t \in[0,1] \\ 0 \leq x, y \leq R}} f_{i}(t, x, y) \leq \phi\left(\frac{R}{L_{1}}\right), \quad i=1,2,
$$

the conclusion of Theorem 3.3 is valid.

Or we choose

$$
\begin{aligned}
\widetilde{L}_{3} & =\varrho \theta \int_{0}^{1} \varphi_{2}^{-1}\left(s^{\beta_{2}-1}\right) s(1-s)^{\alpha_{2}-1} \varphi_{2}^{-1}\left(\int_{0}^{1} G_{2}(1, \tau) d \tau\right) d s, \\
\theta & =\min _{t \in[a, b]}\left\{t^{\alpha_{1}-1}, t^{\alpha_{2}-1}\right\} .
\end{aligned}
$$

Then, for

$$
\lambda_{2} \min _{\substack{t \in[a, b] \subset(0,1) \\ \omega r \leq x, y \leq r}} f_{2}(t, x, y) \geq \phi\left(\frac{r}{\widetilde{L}_{3}}\right), \quad \lambda_{i} \max _{\substack{t \in[0,1] \\ 0 \leq x, y \leq R}} f_{i}(t, x, y) \leq \phi\left(\frac{R}{L_{1}}\right), \quad i=1,2,
$$

the conclusion of Theorem 3.3 is valid.

Theorem 3.4 Assume that $\left(\mathbf{H}_{0}\right)\left(\mathbf{H}_{1}\right)\left(\mathbf{H}_{2}\right)$ hold and $f_{i 0}=f_{i \infty}=+\infty$, then there exists $\lambda_{i}^{*}>0$ such that system (1) has at least two positive solutions for $\lambda_{i} \in\left(0, \lambda_{i}^{*}\right), i=1,2$. 
Proof Choose $r>0$, define

$$
\chi_{i}(r)=\sup _{r>0} \frac{\phi(r)}{\varphi_{2}\left(L_{1}\right) \max _{\substack{t \in[0,1] \\ 0 \leq x, y \leq r}} f_{i}(t, x, y)}, \quad i=1,2 .
$$

In view of the continuity of $f_{i}$ and $f_{i 0}=f_{i \infty}=+\infty$, we know $\chi_{i}(r):(0,+\infty) \rightarrow(0,+\infty)$ is continuous and

$$
\lim _{t \rightarrow 0^{+}} \chi_{i}(r)=\lim _{t \rightarrow+\infty} \chi_{i}(r)=0
$$

So, there exists $r^{*} \in(0,+\infty)$ such that $\chi_{i}\left(r^{*}\right)=\sup _{r>0} \chi_{i}(r)=\lambda_{i}^{*}$. Therefore, for $\lambda_{i} \in\left(0, \lambda_{i}^{*}\right)$, we can find $r_{1}, r_{2}\left(0<r_{1}<r^{*}<r_{2}<+\infty\right)$ satisfying $\chi_{i}\left(r_{1}\right)=\lambda_{1}, \chi_{i}\left(r_{2}\right)=\lambda_{2}$. Thus, by $\left(\mathbf{H}_{0}\right)$, we have

$$
\begin{aligned}
& \lambda_{1} \max _{\substack{t \in[0,1] \\
0 \leq x, y \leq r_{1}}} f_{i}(t, x, y) \leq \frac{\phi\left(r_{1}\right)}{\varphi_{2}\left(L_{1}\right)} \leq \phi\left(\frac{r_{1}}{L_{1}}\right), \\
& \lambda_{2} \max _{\substack{t \in[0,1] \\
0 \leq x, y \leq r_{2}}} f_{i}(t, x, y) \leq \frac{\phi\left(r_{2}\right)}{\varphi_{2}\left(L_{1}\right)} \leq \phi\left(\frac{r_{2}}{L_{1}}\right) .
\end{aligned}
$$

From the condition $f_{i 0}=f_{i \infty}=+\infty$, there exist $R_{1}, R_{2}\left(0<R_{1}<r_{1}<r^{*}<r_{2}<R_{2}<+\infty\right)$ satisfying

$$
\begin{array}{ll}
\frac{f_{1}(t, x, y)}{\phi(x)} \geq \frac{1}{\lambda_{1} \varphi_{1}(\omega \theta) \varphi_{1}\left(L_{3}\right)}, \quad(x, y) \in\left[0, R_{1}\right] \cup\left[R_{2},+\infty\right), t \in[a, b] \subset(0,1), \\
\frac{f_{2}(t, x, y)}{\phi(y)} \geq \frac{1}{\lambda_{2} \varphi_{1}(\omega \theta) \varphi_{1}\left(L_{3}\right)}, \quad(x, y) \in\left[0, R_{1}\right] \cup\left[R_{2},+\infty\right), t \in[a, b] \subset(0,1) .
\end{array}
$$

Hence, by $\left(\mathbf{H}_{0}\right)$, we get

$$
\begin{gathered}
\lambda_{i} \min _{\substack{t \in[a, b] \subset(0,1) \\
\omega \theta R_{1} \leq x, y \leq R_{1}}} f_{i}(t, x, y) \geq \phi\left(\frac{R_{1}}{L_{3}}\right), \\
\lambda_{i} \min _{\substack{t \in[a, b] \subset(0,1) \\
\omega \theta R_{2} \leq x, y \leq R_{2}}} f_{i}(t, x, y) \geq \phi\left(\frac{R_{1}}{L_{3}}\right) .
\end{gathered}
$$

By (37) and (39), (38) and (40), combining with Lemmas 2.6, 2.7 and Theorem 3.3, system (1) has at least two positive solutions for $\lambda_{i} \in\left(0, \lambda_{i}^{*}\right), i=1,2$. The proof is completed.

Remark 3.4 From the proof of Theorem 3.4, assume that $\left(\mathbf{H}_{0}\right)\left(\mathbf{H}_{1}\right)\left(\mathbf{H}_{2}\right)$ hold, if $f_{i 0}=+\infty$ or $f_{i \infty}=+\infty$, then there exists $\lambda_{i}^{*}>0$ such that system (1) has at least one positive solution for $\lambda_{i} \in\left(0, \lambda_{i}^{*}\right), i=1,2$.

\subsection{Nonexistence of system (1)}

Theorem 3.5 Assume that $\left(\mathbf{H}_{0}\right)\left(\mathbf{H}_{1}\right)\left(\mathbf{H}_{2}\right)$ hold and $f_{i}^{\infty}<+\infty, f_{i}^{0}<+\infty$, then there exists $\lambda_{i 0}>0$ such that for $\lambda_{i} \in\left(0, \lambda_{i 0}\right)(i=1,2)$, system (1) has no positive solution. 
Proof From the definitions of $f_{i}^{\infty}, f_{i}^{0}$, which are finite, there exist positive constants $M_{i}^{1}$, $M_{i}^{2}$ and $R_{1}, R_{2}\left(R_{1}<R_{2}\right)$ such that

$$
\begin{array}{ll}
f_{i}(t, x, y) \leq M_{i}^{1} \max \{\phi(x), \phi(y)\}, & 0 \leq x, y \leq R_{1}, t \in[0,1], \\
f_{i}(t, x, y) \leq M_{i}^{2} \max \{\phi(x), \phi(y)\}, & x, y \geq R_{2}, t \in[0,1] .
\end{array}
$$

Set $M_{i}^{0}=\max \left\{M_{i}^{1}, M_{i}^{2}, \max _{t \in[0,1], R_{1} \leq x, y \leq R_{2}} \frac{f_{i}(t, x, y)}{\max \{\phi(x), \phi(y)\}}\right\}$, we have

$$
f_{i}(t, x, y) \leq M_{i}^{0} \max \{\phi(x), \phi(y)\}, \quad x, y \geq 0, t \in[0,1] .
$$

Assume that $(u, v)$ is a positive solution of system (1), we will show that this leads to a contradiction. Define $\lambda_{i 0}=\left(M_{i}^{0}\right)^{-1} \varphi_{1}\left(L_{1}^{-1}\right)$, since $\lambda_{i} \in\left(0, \lambda_{i 0}\right)$, by Lemmas 1.1, 2.3 and 2.5, we conclude that

$$
\begin{aligned}
u(t)= & T_{1}(u, v)(t) \\
= & \int_{0}^{1} K_{1}(t, s) \phi^{-1}\left(\lambda_{1} \int_{0}^{1} G_{1}(s, \tau) f_{1}(\tau, u(\tau), v(\tau)) d \tau\right) d s \\
& +\int_{0}^{1} H_{1}(t, s) \phi^{-1}\left(\lambda_{2} \int_{0}^{1} G_{2}(s, \tau) f_{2}(\tau, u(\tau), v(\tau)) d \tau\right) d s \\
\leq & \int_{0}^{1} \rho t^{\alpha_{1}-1} \phi^{-1}\left(\lambda_{1} \int_{0}^{1} G_{1}(\tau, \tau) M_{1}^{0} \max \{\phi(u(\tau)), \phi(v(\tau))\} d \tau\right) d s \\
& +\int_{0}^{1} \rho t^{\alpha_{1}-1} \phi^{-1}\left(\lambda_{2} \int_{0}^{1} G_{2}(\tau, \tau) M_{2}^{0} \max \{\phi(u(\tau)), \phi(v(\tau))\} d \tau\right) d s \\
\leq & \rho\|(u, v)\| \varphi_{1}^{-1}\left(\lambda_{1} M_{1}^{0}\right) \varphi_{1}^{-1}\left(\int_{0}^{1} G_{1}(\tau, \tau) d \tau\right) \\
& +\rho\|(u, v)\| \varphi_{1}^{-1}\left(\lambda_{2} M_{2}^{0}\right) \varphi_{1}^{-1}\left(\int_{0}^{1} G_{2}(\tau, \tau) d \tau\right) \\
\leq & \|(u, v)\| \varphi_{1}^{-1}\left(\lambda_{1} M_{1}^{0}\right) \frac{L_{1}}{2}+\|(u, v)\| \varphi_{1}^{-1}\left(\lambda_{2} M_{2}^{0}\right) \frac{L_{1}}{2} .
\end{aligned}
$$

Therefore, we conclude

$$
\begin{aligned}
\|u\| & \leq\|(u, v)\| \varphi_{1}^{-1}\left(\lambda_{1} M_{1}^{0}\right) \frac{L_{1}}{2}+\|(u, v)\| \varphi_{1}^{-1}\left(\lambda_{2} M_{2}^{0}\right) \frac{L_{1}}{2} \\
& <\|(u, v)\| \varphi_{1}^{-1}\left(\lambda_{10} M_{1}^{0}\right) \frac{L_{1}}{2}+\|(u, v)\| \varphi_{1}^{-1}\left(\lambda_{20} M_{2}^{0}\right) \frac{L_{1}}{2}=\|(u, v)\| .
\end{aligned}
$$

Similarly to (41) (42), we also have

$$
\|v\|<\|(u, v)\| .
$$

Hence, by (42) (43), we get

$$
\|(u, v)\|=\max \{\|u\|,\|v\|\}<\|(u, v)\|,
$$

which is a contradiction. Therefore, system (1) has no positive solution. The proof is completed. 
Theorem 3.6 Assume that $\left(\mathbf{H}_{0}\right)\left(\mathbf{H}_{1}\right)\left(\mathbf{H}_{2}\right)$ hold and $f_{i \infty}>0, f_{i 0}>0, f_{i}(t, x, y)>0$ for $t \in$ $[a, b] \subset(0,1), x \geq 0, y>0$ or $t \in[a, b] \subset(0,1), x>0, y \geq 0$, then there exists $\lambda_{i *}>0$ such that for $\lambda_{i} \in\left(\lambda_{i *},+\infty\right)(i=1,2)$, system (1) has no positive solution.

Proof From the definitions of $f_{i \infty}, f_{i 0}$, which are finite, there exist positive constants $m_{i}^{1}$, $m_{i}^{2}$ and $R_{3}, R_{4}\left(R_{3}<R_{4}\right)$ such that

$$
\begin{aligned}
& f_{1}(t, x, y) \geq m_{1}^{1} \phi(x), \quad 0 \leq x, y \leq R_{3}, t \in[a, b] \subset(0,1), \\
& f_{1}(t, x, y) \geq m_{1}^{2} \phi(x), \quad x, y \geq R_{4}, t \in[a, b] \subset(0,1) .
\end{aligned}
$$

Set $m_{1}^{0}=\min \left\{m_{1}^{1}, m_{1}^{2}, \min _{t \in[a, b] \subset(0,1), R_{3} \leq x, y \leq R_{4}} \frac{f_{1}(t, x, y)}{\phi(x)}\right\}$, we have

$$
f_{1}(t, x, y) \geq m_{1}^{0} \phi(x), \quad x, y \geq 0, t \in[a, b] \subset(0,1) .
$$

Similarly, set $m_{2}^{0}=\min \left\{m_{2}^{1}, m_{2}^{2}, \min _{t \in[a, b] \subset(0,1), R_{3} \leq x, y \leq R_{4}} \frac{f_{2}(t, x, y)}{\phi(y)}\right\}$, we have

$$
f_{2}(t, x, y) \geq m_{2}^{0} \phi(y), \quad x, y \geq 0, t \in[a, b] \subset(0,1) .
$$

Assume that $(u, v)$ is a positive solution of system (1), we will show that this leads to a contradiction. Define $\lambda_{i *}=\left(m_{i}^{0}\right)^{-1} \varphi_{2}\left(L_{2}^{-1}\right)$, since $\lambda_{i} \in\left(\lambda_{i *},+\infty\right)$, by Lemmas 1.1, 2.3 and 2.5, we conclude that

$$
\begin{aligned}
\|u\|= & \left\|T_{1}(u, v)\right\| \\
\geq & \min _{t \in[a, b]} \int_{0}^{1} \varrho t^{\alpha_{1}-1} s(1-s)^{\alpha_{1}-1} \phi^{-1}\left(\lambda_{1} \int_{0}^{1} s^{\beta_{1}-1} G_{1}(1, \tau) m_{1}^{0} \phi(u(\tau)) d \tau\right) d s \\
& +\int_{0}^{1} \varrho t^{\alpha_{1}-1} s(1-s)^{\alpha_{2}-1} \phi^{-1}\left(\lambda_{2} \int_{0}^{1} s^{\beta_{2}-1} G_{2}(1, \tau) m_{2}^{0} \phi(v(\tau)) d \tau\right) d s \\
\geq & \varrho \omega \theta^{2}\|(u, v)\| \int_{0}^{1} \varphi_{2}^{-1}\left(s^{\beta_{1}-1}\right) s^{\beta_{1}-1} \varphi_{2}^{-1}\left(\int_{0}^{1} G_{1}(1, \tau) d \tau\right) d s \psi_{2}^{-1}\left(\lambda_{1} m_{1}^{0}\right) \\
& +\varrho \omega \theta^{2}\|(u, v)\| \int_{0}^{1} \varphi_{2}^{-1}\left(s^{\beta_{2}-1}\right) s^{\beta_{2}-1} \varphi_{2}^{-1}\left(\int_{0}^{1} G_{2}(1, \tau) d \tau\right) d s \psi_{2}^{-1}\left(\lambda_{2} m_{2}^{0}\right) \\
\geq & \|(u, v)\| \varphi_{2}^{-1}\left(\lambda_{1} m_{1}^{0}\right) \frac{L_{2}}{2}+\|(u, v)\| \varphi_{2}^{-1}\left(\lambda_{2} m_{2}^{0}\right) \frac{L_{2}}{2}>\|(u, v)\| .
\end{aligned}
$$

Similarly to (45), we also have

$$
\|v\|>\|(u, v)\| .
$$

Hence, by (45) (46), we get

$$
\|(u, v)\|=\max \{\|u\|,\|v\|\}>\|(u, v)\|,
$$

which is a contradiction. Therefore, system (1) has no positive solution. The proof is completed.

Similar to the proof of Theorem 3.6, we obtain the following Theorems 3.7 and 3.8. 
Theorem 3.7 Assume that $\left(\mathbf{H}_{0}\right)\left(\mathbf{H}_{1}\right)\left(\mathbf{H}_{2}\right)$ hold and $f_{1 \infty}>0, f_{10}>0, f_{1}(t, x, y)>0$ for $t \in$ $[a, b] \subset(0,1), x \geq 0, y>0$ or $t \in[a, b] \subset(0,1), x>0, y \geq 0$, then there exists $\lambda_{1 *}>0$ such that for $\lambda_{1} \in\left(\lambda_{1 *},+\infty\right), \lambda_{2} \in(0,+\infty)$, system (1) has no positive solution.

Theorem 3.8 Assume that $\left(\mathbf{H}_{0}\right)\left(\mathbf{H}_{1}\right)\left(\mathbf{H}_{2}\right)$ hold and $f_{2 \infty}>0, f_{20}>0, f_{2}(t, x, y)>0$ for $t \in$ $[a, b] \subset(0,1), x \geq 0, y>0$ or $t \in[a, b] \subset(0,1), x>0, y \geq 0$, then there exists $\lambda_{2 *}>0$ such that for $\lambda_{2} \in\left(\lambda_{2 *},+\infty\right), \lambda_{1} \in(0,+\infty)$, system (1) has no positive solution.

Remark 3.5 From the proof of Theorems 3.1-3.8, if we choose

$$
\begin{array}{ll}
f_{10}=\liminf _{y \rightarrow 0^{+}} \inf _{\substack{t \in[a, b] \subset(0,1) \\
x \in[0,+\infty)}} \frac{f_{1}(t, x, y)}{\phi(y)}, & f_{1}^{0}=\limsup _{y \rightarrow 0^{+}} \sup _{\substack{t \in[0,1] \\
x \in[0,+\infty)}} \frac{f_{1}(t, x, y)}{\phi(y)}, \\
f_{20}=\liminf _{x \rightarrow 0^{+}} \inf _{\substack{t \in[a, b] \subset(0,1) \\
y \in[0,+\infty)}} \frac{f_{2}(t, x, y)}{\phi(x)}, & f_{2}^{0}=\limsup _{x \rightarrow 0^{+}} \sup _{\substack{t \in[0,1] \\
y \in[0,+\infty)}} \frac{f_{2}(t, x, y)}{\phi(x)}, \\
f_{1 \infty}=\liminf _{y \rightarrow+\infty} \inf _{\substack{t \in[a, b] \subset(0,1) \\
x \in[0,+\infty)}} \frac{f_{1}(t, x, y)}{\phi(y)}, & f_{1}^{\infty}=\limsup _{y \rightarrow+\infty} \sup _{\substack{t \in[0,1] \\
x \in[0,+\infty)}} \frac{f_{1}(t, x, y)}{\phi(y)}, \\
f_{2 \infty}=\liminf _{x \rightarrow+\infty} \inf _{\substack{t \in[a, b] \subset(0,1) \\
y \in[0,+\infty)}} \frac{f_{2}(t, x, y)}{\phi(x)}, & f_{2}^{\infty}=\limsup _{x \rightarrow+\infty} \sup _{\substack{t \in[0,1] \\
y \in[0,+\infty)}} \frac{f_{2}(t, x, y)}{\phi(x)},
\end{array}
$$

then all the conclusion of Theorems 3.1-3.8 are valid.

\section{Example}

Consider the fractional differential system

$$
\left\{\begin{array}{l}
D^{\frac{3}{2}}\left(D_{0^{+}}^{\frac{5}{2}} u(t)\right)+\lambda_{1} f_{1}(t, u(t), v(t))=0, \\
D^{\frac{3}{2}}\left(D_{0^{+}}^{\frac{5}{2}} v(t)\right)+\lambda_{2} f_{2}(t, u(t), v(t))=0, \quad 0<t<1, \\
u(0)=u^{\prime}(0)=0, \quad D_{0^{+}}^{\frac{5}{2}} u(0)=\left(D_{0^{+}}^{\frac{5}{2}} u(1)\right)^{\prime}=0, \quad u(1)=\frac{1}{2} \int_{0}^{1} t^{-\frac{1}{2}} v(t) d t, \\
v(0)=v^{\prime}(0)=0, \quad D_{0^{+}}^{\frac{5}{2}} v(0)=\left(D_{0^{+}}^{\frac{5}{2}} v(1)\right)^{\prime}=0, \quad v(1)=\int_{0}^{1} u(t) d t^{\frac{1}{2}},
\end{array}\right.
$$

where $\lambda_{i}>0(i=1,2)$ is a parameter, $\alpha_{1}=\alpha_{2}=\frac{5}{2}, \beta_{1}=\beta_{2}=\frac{3}{2}, \mu_{1}=\frac{1}{2}, \mu_{2}=1, A_{1}(t)=t$, $A_{2}(t)=t^{\frac{1}{2}}, g_{1}(t)=t^{-\frac{1}{2}}, g_{2}(t)=1, \phi(x)=x$, choose $\varphi_{1}(x)=\varphi_{2}(x)=x$. Then we have

$$
\begin{aligned}
& k_{1}=\int_{0}^{1} g_{1}(t) t^{\alpha_{2}-1} d A_{1}(t)=\int_{0}^{1} t^{-\frac{1}{2}} t^{\frac{3}{2}} d t=\frac{1}{2}>0, \\
& k_{2}=\int_{0}^{1} g_{2}(t) t^{\alpha_{1}-1} d A_{2}(t)=\int_{0}^{1} t^{\frac{3}{2}} d t^{\frac{1}{2}}=\frac{1}{2} \int_{0}^{1} t d t=\frac{1}{4}>0, \\
& 1-\mu_{1} \mu_{2} k_{1} k_{2}=\frac{15}{16}>0 .
\end{aligned}
$$

So, condition $\left(\mathbf{H}_{1}\right)$ holds. Next, in order to demonstrate the application of our main results obtained in Section 3, we choose two different sets of functions $f_{i}(i=1,2)$ such that $f_{i}$ satisfies the conditions of Theorems 3.1 and 3.5.

Case 1. Let $f_{1}(t, x, y)=\frac{x^{2}}{1+t}+x \sin y, f_{2}(t, x, y)=\frac{y^{2}}{e^{t}}+y \sin x$, choose $\left[\frac{1}{4}, \frac{2}{2}\right] \subset[0,1]$, we know $f_{i \infty}=+\infty, f_{i}^{0}=0$. Then, by Theorem 3.1, system (48) has at least one positive solution for $\lambda_{i} \in(0,+\infty)(i=1,2)$. 
Case 2. Let $f_{1}(t, x, y)=\frac{\left(10 x^{2}+x\right)(3+\sin y)}{(1+t)(x+1)}, f_{2}(t, x, y)=\frac{\left(10 y^{2}+y\right)(2+\sin x)}{e^{t}(y+1)}$, therefore, we have $f_{1}^{\infty}=40$, $f_{1}^{0}=3, f_{2}^{\infty}=30, f_{2}^{0}=2$, and for $x, y \leq 0$, we get $x \leq f_{1}(t, x, y) \leq 40 x, y \leq f_{2}(t, x, y) \leq 30 y$. By calculation, we obtain $L_{1}=2.2445 \int_{0}^{1} \frac{\tau^{\frac{1}{2}}(1-\tau)^{-\frac{1}{2}}}{\Gamma\left(\frac{3}{2}\right)} d \tau \approx 0.6632$. Then, by Theorem 3.5, system (48) has no positive solution for $\lambda_{1} \in(0,0.0377), \lambda_{2} \in(0,0.0503)$.

\section{Acknowledgements}

The authors were supported financially by the National Natural Science Foundation of China $(11626125,11701252)$, Natural Science Foundation of Shandong Province of China (ZR2016AP04), China Postdoctoral Science Foundation funded project (2017M612231), a Project of Shandong Province Higher Educational Science and Technology Program (J16LI03), the Science Research Foundation for Doctoral Authorities of Linyi University (LYDX2016BS080), and the Applied Mathematics Enhancement Program of Linyi University.

\section{Competing interests}

The authors declare that they have no competing interests.

\section{Authors' contributions}

All authors contributed equally to the writing of this paper. All authors read and approved the final manuscript.

\section{Author details}

${ }^{1}$ School of Mathematics and Statistics, Linyi University, Linyi, Shandong 276000, People's Republic of China. ${ }^{2}$ Key Laboratory of Complex Systems and Intelligent Computing, Universities of Shandong (Linyi University), Linyi, Shandong 276000, People's Republic of China. ${ }^{3}$ School of Mathematical Sciences, Qufu Normal University, Qufu, Shandong 273165 , People's Republic of China.

\section{Publisher's Note}

Springer Nature remains neutral with regard to jurisdictional claims in published maps and institutional affiliations.

Received: 5 July 2017 Accepted: 2 October 2017 Published online: 23 October 2017

\section{References}

1. Wang, H: On the number of positive solutions of nonlinear systems. J. Math. Anal. Appl. 281, 287-306 (2003)

2. Leibenson, LS: General problem of the movement of a compressible fluid in a porous medium. Izv. Akad. Nauk SSSR, Ser. Geogr. Geofiz. IX, 7-10 (1983) (in Russian)

3. Murray, JD: Mathematical Biology. Springer, New York (1993)

4. Lu, HL, Han, ZL, Sun, SR, Liu, J: Existence on positive solutions for boundary value problems of nonlinear fractional differential equations with p-Laplacian. Adv. Differ. Equ. 2013, 30 (2013)

5. Wang, J, Xiang, H, Liu, Z: Positive solutions for three-point boundary value problems of nonlinear fractional differential equations with p-Laplacian. Far East J. Appl. Math. 37, 33-47 (2009)

6. Agarwal, RP, O'Regan, D, Papageorgiun, NS: On the existence of two nontrivial solutions of periodic problems with operators of p-Laplacian type. Differ. Equ. 43, 157-163 (2007)

7. Zhang, XG, Liu, LS, Wiwatanapataphee, B, Wu, YH: The eigenvalue for a class of singular $p$-Laplacian fractional differential equations involving the Riemann-Stieltjes integral boundary condition. Appl. Math. Comput. 235, 412-422 (2014)

8. Mahmudov, NI, Unul, S: Existence of solutions of fractional boundary value problems with $p$-Laplacian operator. Bound. Value Probl. 2015, 99 (2015)

9. Liu, LS, Zhang, XQ, Jiang, J, Wu, YH: The unique solution of a class of sum mixed monotone operator equations and its application to fractional boundary value problems. J. Nonlinear Sci. Appl. 9, 2943-2958 (2016)

10. Sun, FL, Liu, LS, Zhang, XG, Wu, YH: Spectral analysis for a singular differential system with integral boundary conditions. Mediterr. J. Math. 13, 4763-4782 (2016)

11. Hao, XA, Liu, LS, Wu, YH: Positive solutions for nonlinear fractional semipositone differential equation with nonlocal boundary conditions. J. Nonlinear Sci. Appl. 9, 3992-4002 (2016)

12. Hao, XA, Liu, LS: Mild solution of semilinear impulsive integro-differential evolution equation in Banach spaces. Math. Methods Appl. Sci. 40, 4832-4841 (2017)

13. Wang, Y, Liu, LS: Uniqueness and existence of positive solutions for the fractional integro-differential equation. Bound. Value Probl. 2017, 12 (2017)

14. Wang, Y, Li, J, Cai, ZX: Positive solutions of periodic boundary value problems for the second-order differential equation with a parameter. Bound. Value Probl. 2017, 49 (2017)

15. Liu, LS, Sun, FL, Zhang, XG, Wu, YH: Bifurcation analysis for a singular differential system with two parameters via to degree theory. Nonlinear Anal., Model. Control 22(1), 31-50 (2017)

16. Henderson, J, Luca, R: Positive solutions for a system of nonlocal fractional boundary value problems. Fract. Calc. Appl. Anal. 16(4), 985-1008 (2013)

17. Wang, JH, Xiang, HJ, Liu, ZG: Positive solution to nonzero boundary values problem for a coupled system of nonlinear fractional differential equations. Int. J. Differ. Equ. 2010, Article ID 186928 (2010)

18. Wang, GT, Agarwal, RP, Cabada, A: Existence results and the monotone iterative technique for systems of nonlinear fractional differential equations. Appl. Math. Comput. 25, 1019-1024 (2012)

19. Henderson, J, Luca, R: Positive solutions for a system of fractional differential equations with coupled integral boundary conditions. Appl. Math. Comput. 249, 182-197 (2014) 
20. Goodrich, CS: Existence of a positive solution to systems of differential equations of fractional order. Comput. Math. Appl. 62, 1251-1268 (2011)

21. Yang, WG: Positive solutions for a coupled system of nonlinear fractional differential equations with integral boundary conditions. Comput. Math. Appl. 63, 288-297 (2012)

22. Wang, Y, Liu, LS, Wu, YH: Positive solutions for a class of higher-order singular semipositone fractional differential systems with coupled integral boundary conditions and parameters. Adv. Differ. Equ. 2014, 268 (2014)

23. Wang, Y, Liu, LS, Wu, YH: Positive solutions for $(n-1,1)$-type singular fractional differential system with coupled integral boundary conditions. Abstr. Appl. Anal. 2014, Article ID 142391 (2014)

24. Miller, KS, Ross, B: An Introduction to the Fractional Calculus and Fractional Differential Equations. Wiley, New York (1993)

25. Podlubny, I: Fractional Differential Equations. Mathematics in Science and Engineering, vol. 198. Academic Press, New York (1999)

26. El-Shahed, M: Positive solutions for boundary value problem of nonlinear fractional differential equation. Abstr. Appl. Anal. 2007, Article ID 10368 (2007)

27. Guo, DJ, Lakshmikantham, V: Nonlinear Problems in Abstract Cones. Academic Press, New York (1988)

Submit your manuscript to a SpringerOpen ${ }^{\circ}$ journal and benefit from:

- Convenient online submission

- Rigorous peer review

- Open access: articles freely available online

- High visibility within the field

- Retaining the copyright to your article

Submit your next manuscript at $\boldsymbol{\nabla}$ springeropen.com 